\title{
Carbapenem Resistant Acinetobacter
}

National Cancer Institute

\section{Source}

National Cancer Institute. Carbapenem Resistant Acinetobacter. NCI Thesaurus. Code C150879.

Any species, subspecies, or strain of Acinetobacter that is able to grow in the presence of carbapenem. 